\title{
The effect of the use of smartphone while walking on the electromyography activity of the lower extremity in young students
}

\author{
Dae-Hee Lee, Hye-Joo Jeon* \\ Department of Physical Therapy, U1 University, Yeongdong, Korea
}

The study aims to determine the effects of smartphone use on the muscle activity of the lower extremity when walking. Twenty-three healthy young students were asked to perform a 10-m walk test between normal walking without using a smartphone and walking while two-handed texting on a smartphone. The electromyography activities of the lower extremity were quantified. To quantitatively assess the cervical flexion range of smartphone users, the cervical flexion angle was measured using a digital goniometer. The study results indicated that the use of a smartphone while walking could lessen muscle activity on the tibialis anterior, gastrocnemius, rectus femoris, gluteus maximus, and gluteus medius than that of normal walking without using a smartphone. The walking speeds were reduced in walking while using a smartphone compared with normal walking without using a smartphone. The cervical flexion angle was greater when walking while using a smartphone compared to that of normal walking without using a smartphone. These results suggest that frequently using a smartphone while walking could be a potential risk for musculoskeletal problems.

Keywords: Electromyography, Muscle activity, Walking, Smartphone, Lower extremity

\section{INTRODUCTION}

Walking has been associated with many advantages for people's well-being and physical health (Abraham et al., 2010). Understanding individual muscle forces during walking can help to better identify several musculoskeletal injuries from gait analysis and provide a better insight on how these affect walking (Lin and Huang, 2017).

Electromyography (EMG) has been used to monitor the electrical activity in the lower limb muscles (Alghamdi and Preece, 2020). Some studies have researched how muscles contribute to support and the progression during normal gait (Anderson and Pandy, 2003; Liu et al., 2006; Neptune et al., 2001, 2004). Neptune et al. (2008) reported that the gluteus maximus, vasti, soleus, and gastrocnemius were influential for trunk support over a range of walking speeds.

Smartphones are considered a necessary part of the daily life in

${ }^{*}$ Corresponding author: Hye-Joo Jeon (DD https://orcid.org/0000-0001-8491-3074 Department of Physical Therapy, U1 University, 310 Daehak-ro, Yeongdong-eup, Yeongdong-gun, Chungcheongbuk-do 29131, Korea Email: bluestar@ul.ac.kr

Received: March 9, 2021 / Accepted: March 26, 2021 all age groups. An estimated $77 \%$ of the world's population owns a smartphone, where texting emerged as a quick and cost-effective communication method (Schabrun et al., 2014). In a study conducted from 2014 to 2015, 2,367 students from the Saudi University participated. Of these 2,367 students, $27.2 \%$ admitted using their smartphones for more than $8 \mathrm{hr}$ per day and $75 \%$ of them admitted using it for 4 hr a day (Alosaimi et al., 2016). Excessive smartphone use is associated with repetitive strain on joint, resulting in inflammatory changes in healthy joints (Megna et al., 2018). According to a research, $82 \%$ of the smartphone users experienced pain in the back, shoulder, neck, and hands, with greater severity associated with more time spent on their devices (Berolo et al., 2011). In cities, human behavior when walking has changed greatly: many pedestrians now use their smartphones while walking (Basch et al., 2015). Nasar and Troyer (2013) reported a 6-fold increase in the incidence of smartphone-related pedestrian injuries from 2005 to 2010. Given the increasing rate of smartphone use, 
it is unlikely that this trend will reverse without countermeasure. Using a smartphone while crossing a virtual street increased the time spent looking away from the road and the frequency of car crash (Byington and Schwebel, 2013). It may alter walking as a result of the increased cognitive demand placed on the working memory and the executive control during dual-task performance (Rubinstein et al., 2001).

A previous research has shown that using a smartphone while walking leads to changes in gait, characterized by decreased step length, increased step width, longer standing on each limb, and a decrease in swing time (Parr et al., 2014). In another study, researchers measured severity of the head-forward tilt posture of smartphone use and found that those using a smartphone while sitting and standing tend to put their heads down (Lee et al., 2015). The head-down posture when using smartphones contributes to the occurrence of neck musculoskeletal disorder (Berolo et al., 2011). The pain occurs in the neck and other body parts, including the wrists, hands, thumbs, and fingers (Barr et al., 2004; Gustafsson et al., 2010; Ming et al., 2006).

Recently, healthy young individuals walked with more thoracic kyphosis and lumbar lordosis when using a smartphone compared to when walking without using a smartphone. The results show that the routine use of a smartphone while walking could be a risk for low back pain and musculoskeletal disorders (Choi et al., 2021). Betsch et al. (2021) investigated that the excessive smartphone use can lead to major changes in the spinal posture, such as increased trunk inclination and thoracic kyphosis while standing and walking.

However, no study has yet quantified the EMG of the lower extremity muscles when using a smartphone while walking. To further explore the effects of smartphone use on walking, we examined and compared the EMG amplitudes of the lower extremity muscles during normal walking without using a smartphone and texting on a smartphone while walking. We hypothesized that walking while using a smartphone would change the lower extremity muscle activity compared to normal walking.

Table 1. General characteristics of the subjects $(n=23)$

\begin{tabular}{lc}
\hline Variable & Value \\
\hline Age $(\mathrm{yr})$ & $24.51 \pm 2.68$ \\
Height $(\mathrm{cm})$ & $169.10 \pm 8.73$ \\
Weight $(\mathrm{kg})$ & $70.28 \pm 13.54$ \\
Gender, male:femal & 14.9 \\
Duration of use of the smartphone per day (min) & $329.13 \pm 61.92$
\end{tabular}

Values are presented as mean \pm standard deviation or number.

\section{METERIALS AND METHODS}

\section{Participants}

Twenty-three healthy young students (14 males, 9 females) were recruited from the university community, with the inclusion criteria of belonging to the study target. The exclusion criteria were having a history of fracture of the pelvis and the lower extremity, diseases affecting walking, and not understanding the evaluator's commands. In our survey, it was reported that the participants spend an average of $329.13 \pm 61.92 \mathrm{~min}$ per day using smartphones. The subject characteristics are presented in Table 1. This study was approved by the Ethics Committee of U1 University (approval number: 2020-07). All subjects provided written informed consent.

\section{Methods}

Each participant walked flat on the ground in two different conditions: normal walking without using a smartphone and walking while conducting two-handed texting on a smartphone. The subjects were asked to text the first verse of the national anthem. After shaving, debriding, and cleaning the skin surface, electrodes were placed over the following eight right lower limb muscles: gluteus maximus and medius, biceps femoris, rectus femoris, gastrocnemius (medial and lateral parts), tibialis anterior, and soleus. With a tape, the surface electrodes were positioned longitudinally over the abdominal muscle, approximately in the direction of the muscle fibers, according to the SENIAM recommendations (Table 2) (Hermens et al., 2000). While conducting the walks, the EMG signals from the muscles were collected using a surface EMG system.

Table 2. Electrode location descriptions on the muscle

\begin{tabular}{|c|c|}
\hline Muscle & Electrode location \\
\hline Gluteus maximus & $\begin{array}{l}\text { At } 50 \% \text { on the line between the sacral vertebrae and } \\
\text { the greater trochanter }\end{array}$ \\
\hline Gluteus medius & At $50 \%$ on the line from the crista iliaca to the trochanter \\
\hline Biceps femoris & $\begin{array}{l}\text { At } 50 \% \text { on the line between the ischial tuberosity and } \\
\text { the lateral epicondyle of the tibia }\end{array}$ \\
\hline Rectus femoris & $\begin{array}{l}\text { At } 50 \% \text { on the line from the anterior spina iliaca superior } \\
\text { to the superior part of the patella }\end{array}$ \\
\hline Gastrocnemius (medial) & On the most prominent bulge of the muscle \\
\hline Gastrocnemius (lateral) & $\begin{array}{l}\text { At } 1 / 3 \text { of the line between the head of the fibula and the } \\
\text { heel }\end{array}$ \\
\hline Tibialis anterior & $\begin{array}{l}\text { At } 1 / 3 \text { on the line between the tip of the fibula and the } \\
\text { tip of the medial malleolus }\end{array}$ \\
\hline Soleus & $\begin{array}{l}\text { At } 2 / 3 \text { of the line between the medial condylis of the } \\
\text { femur to the medial malleolus }\end{array}$ \\
\hline
\end{tabular}




\section{Electromyography}

To acquire EMG signals, the surface EMG data were recorded using a Trigno wireless EMG system (Delsys, Inc., Boston, MA, USA). Data analysis was performed using the EMGworks software package (ver. 4.0; Delsys). The sampling rate for the EMG signal was set at 2,000 Hz and the band-pass filter between 20 and $450 \mathrm{~Hz}$. The raw data from the muscles were transformed into the root mean square data. A reference voluntary contraction (RVC) was used to normalize EMG data (Lehman and McGill, 1999). The mean values for the RVC were calculated from each muscle when the subjects were in a static standing for $10 \mathrm{sec}$.

\section{0-Meter walk test}

The 10-m walk test (10-MWT) is a simple assessment to measure walking ability in clinical settings, where an individual independently walked a $14-\mathrm{m}$ distance. The time taken for the intermediate $10 \mathrm{~m}$ is measured, allowing acceleration and deceleration. It can be either tested at the preferred walking speed.

\section{Digital goniometer}

The cervical flexion angle in the sagittal plane was measured from each participant while walking and texting on a smartphone and while normal walking without using a smartphone after 10MWT immediately. The digital goniometer axis was placed at the external auditory meatus and the stationary arm perpendicular to floor. Finally, the moving arm was placed at the base of the nostrils. In the 0 starting position for measuring the cervical flexion range of motion, the goniometer reads $90^{\circ}$, which should be transposed and recorded as $0^{\circ}$.

\section{Statistical analysis}

Statistical analyses were performed using the IBM SPSS ver.

Table 3. Comparison of muscle activity

\begin{tabular}{lccl}
\hline Muscle (\%RVC) & $\begin{array}{c}\text { Normal walking without } \\
\text { using a smartphone }\end{array}$ & $\begin{array}{c}\text { Walking while } \\
\text { using a smartphone }\end{array}$ & $P$-value \\
\hline Tibialis anterior & $689.07 \pm 441.75$ & $595.08 \pm 400.86$ & $0.01^{*}$ \\
Soleus & $1,704.35 \pm 5,863.38$ & $1,973.00 \pm 7,461.34$ & 0.06 \\
Gastrocnemius (medial) & $1,040.37 \pm 1,127.66$ & $888.10 \pm 988.52$ & $0.01^{*}$ \\
Gastrocnemius (lateral) & $650.72 \pm 504.10$ & $494.05 \pm 363.58$ & $0.00^{*}$ \\
Rectus femoris & $785.53 \pm 960.66$ & $695.36 \pm 989.98$ & $0.00^{*}$ \\
Biceps femoris & $1,906.61 \pm 3,444.23$ & $1,455.29 \pm 2,315.15$ & 0.06 \\
Gluteus maximus & $1,398.78 \pm 2,730.48$ & $930.33 \pm 1,943.44$ & $0.01^{*}$ \\
Gluteus medius & $1,201.03 \pm 3,745.77$ & $950.49 \pm 3,257.50$ & $0.00^{*}$ \\
\hline
\end{tabular}

Values are presented as mean \pm standard deviation.

$\mathrm{RVC}$, reference voluntary contraction.

${ }^{*} P<0.05$.
18.0 (IBM Co., Armonk, NY, USA), where the data were expressed as means \pm standard deviations. The differences between normal walking without using a smartphone and walking while texting on a smartphone were assessed by performing the Wilcoxon signedrank test, considering a $P$-value of $<0.05$ as statistically significant. In all measurements in the data, the average value of the three repetitions was calculated.

\section{RESULTS}

\section{Electromyography}

For walking without using a smartphone and walking while using a smartphone, the mean normalized EMG values of the tibialis anterior were $689.07 \% \pm 441.75 \%$ and $595.08 \% \pm 400.86 \%$, medial gastrocnemius $1,040.37 \% \pm 1,127.66 \%$ and $888.10 \% \pm$ $988.52 \%$, lateral gastrocnemius $650.72 \% \pm 504.10 \%$ and $494.05 \% \pm 363.58 \%$, rectus femoris $785.53 \% \pm 960.66 \%$ and $695.36 \% \pm 989.98 \%$, gluteus maximus $1,398.78 \% \pm 2,730.48 \%$ and $930.33 \% \pm 1,943.44 \%$, and gluteus medius $1,201.03 \% \pm$ $3,745.77 \%$, and $950.49 \% \pm 3,257.50 \%$, respectively, showing a significant difference between the two walking tasks $(P<0.05)$. The mean normalized EMG of the biceps femoris was lesser during walking while using a smartphone $(1,455.29 \% \pm 2,315.15 \%)$ than that during normal walking without using a smartphone $(1,906.61 \% \pm 3,444.23 \%)$, having no significant differences $(P>$ $0.05)$. The participants used the soleus muscles more during walking while using a smartphone $(1,973.66 \% \pm 7,461.34 \%)$ than during normal walking without using a smartphone $(1,704.35 \% \pm$ $5,863.38 \%)$, showing no significant differences $(P>0.05)$ (Table 3 ).

\section{0-MWT}

The walking speed was slower during walking while using a smartphone $(10.15 \pm 1.29 \mathrm{sec})$ than that during normal walking without using a smartphone $(8.92 \pm 1.10 \mathrm{sec})(P<0.05)$ (Table 4$)$.

\section{Digital goniometer}

The cervical flexion angle was greater during walking while using a smartphone $\left(30.79^{\circ} \pm 8.63^{\circ}\right)$ than that during normal walk-

Table 4. Comparison of the 10-meter walk test

\begin{tabular}{lccc}
\hline Index & $\begin{array}{c}\text { Normal walking without } \\
\text { using a smartphone }\end{array}$ & $\begin{array}{c}\text { Walking while } \\
\text { using a smartphone }\end{array}$ & $P$-value \\
\hline Speed (sec) & $8.92 \pm 1.10$ & $10.15 \pm 1.29$ & $0.00^{*}$ \\
\hline $\begin{array}{l}\text { Values are presented as mean } \pm \text { standard deviation. } \\
{ }^{*} P<0.05 .\end{array}$
\end{tabular}


Table 5. Comparison of the cervical angle

\begin{tabular}{lccc}
\hline Index & $\begin{array}{c}\text { Normal walking without } \\
\text { using a smartphone }\end{array}$ & $\begin{array}{c}\text { Walking while } \\
\text { using a smartphone }\end{array}$ & $\begin{array}{c}P \text {-value } \\
\text { Cervical flexion angle }\left({ }^{\circ}\right)\end{array}$ \\
\hline
\end{tabular}

Values are presented as mean \pm standard deviation.

${ }^{*} P<0.05$.

ing without using a smartphone $\left(4.27^{\circ} \pm 3.28^{\circ}\right)(P<0.05)$ (Table 5).

\section{DISCUSSION}

The study aims to determine the effects of smartphone use on the lower extremity muscle activity during walking. Our results show that the participants walked with less muscle activity in the gluteus maximus and medius, biceps femoris, rectus femoris, gastrocnemius, and tibialis anterior when using a smartphone compared to when walking without using a smartphone. Researchers found that the vasti, hamstring, gluteus maximus and medius, soleus, gastrocnemius, and dorsiflexors were the central factors of the fore-aft and vertical ground reaction force or the center of mass accelerations while walking (Liu et al., 2006; Neptune et al., 2004). Neptune et al. (2008) reported that the vertical support of the trunk was provided by the vasti, gluteus maximus, gastrocnemius, and soleus, while the forward propulsion of the trunk was provided by the rectus femoris and soleus.

Previous studies analyzed that the hip and knee extensor muscles provide much of the support in the first half of the stance phase (Kepple et al., 1997; Neptune et al., 2004; Pandy, 2001; Winter, 1980). During the early stance, the quadriceps femoris muscle slows the forward progression and affords vertical support (Liu et al., 2006, 2008; Neptune et al., 2004). The gluteus medius is considered the major hip abductor (Flack et al., 2012), with a main function of weight bearing for pelvic stability in the unilateral stance phase against the effects of gravity (Al-Hayani, 2009; Gottschalk et al., 1989). Also, it makes considerable contributions to the vertical acceleration in the single limb support (Anderson and Pandy, 2003).

In the human body, the gluteus maximus is the largest muscle (Ward et al., 2009), which arises from the back bone of the pelvis, attached to the side and back of the upper femur. Its action is hip extension, performing as the major propulsive muscle during upright walking (Lovejoy, 1988). The muscle functions to control the flexion of the trunk relative to the stance limb during walking (Anderson and Pandy, 2003). Interestingly, the human walking economy is a shorter and more dorsally projecting ischium, en- abling hip extensor muscle torque production at $180^{\circ}$ full extension of the hip (Lewton and Scott, 2017; Stern and Susman, 1983). Because of this feature, the straight-legged gait advances walking economy compared to nonhuman apes by decreasing the muscle activity needed to support the body weight in each step (Pontzer et al., 2009; Sockol et al., 2007).

During human walking, the tibialis anterior absorbs energy across the ankle joint as the foot comes in contact with the ground, producing force during the ankle plantar flexes (Usherwood et al., 2012) and plays a major role in controlling foot position and ensuring toe clearance. It is active at the end of the stance phase and into the early swing phase, to help power the ankle dorsiflexion (Hof et al., 2002). The gastrocnemius is important in stair climbing, walking, and running and, together with the soleus, accounts for about $93 \%$ of the plantar flexion torque (Haxton, 1944). The soleus is a one-joint muscle running from the back surface of the fibula and tibia to the Achilles tendon. And its activations are during the end part of the loading response phase, working until the end of the terminal stance phase. The gastrocnemius is a twojoint muscle with two heads located from the medial and lateral condyles of the femur (Park et al., 2019). In gait cycle, the gastrocnemius is active between $10 \%$ and $60 \%$ and contributes in producing energy between $40 \%$ and $60 \%$ when the ground reaction force is directed forward and upward (Nadeau et al., 1999). Eventually, the decrease in gastrocnemius muscle activity while walking using a smartphone would cause difficulty in producing proper energy for walking.

Our study results showed that contrary to the gastrocnemius, the soleus muscle more while walking using a smartphone than in normal walking without using a smartphone, but not statistically significant. There is sufficient evidence showing that decreased medial gastrocnemius force generation would require an increase in soleus activation to maintain enough ankle joint torque (Cronin et al., 2011; Lichtwark and Wilson, 2008). In the present study, the walking speed evaluation revealed that individuals walk slower when walking while texting than normal walking. Researchers indicated that walking speed decreased when texting on a smartphone, which they explained to be caused by the cognitive distraction of a dual task (Lamberg and Muratori, 2012).

Our results showed that the cervical flexion angles were $4.27^{\circ}$ and $30.79^{\circ}$ for walking without using smartphone and walking while using a smartphone, respectively, with a significant difference between the two tasks. This finding was consistent with the earlier result, where the mean cervical flexion angle was $38.5^{\circ}$ for walking while texting on a smartphone. They reported that the use of 
a smartphone while walking poses potential risks for neck musculoskeletal problems due to a large neck flexion (Han and Shin, 2019). The neck flexion increases the moment arm of the head with respect to the rotation axis at the cervical level, and it involves the contraction forces of the neck extensor muscles to support the head weight in the flexed neck (Straker et al., 2008). The increased neck flexion while using a smartphone can have major effects on the entire spine since it is connected in a kinematic chain of joints. Moreover, greater head motion may reduce the vestibular information. The vestibular input is essential for navigation during walking ( $\mathrm{St}$ George and Fitzpatrick, 2011). In an earlier study, the neck flexion angle has been quantified as a potential risk factor leading to biomechanical stress at the cervical level when using a tablet (Douglas and Gallagher, 2017; Young et al., 2012).

A recent study found that healthy young individuals walked with more thoracic kyphosis and lumbar lordosis when using a smartphone compared to when walking without using a smartphone (Choi et al., 2021). Additionally, walking while using a smartphone may slightly increase the pelvic anterior tilting, knee hyperextension, and ankle plantar flexion, thus, rendering less muscle activity of the gluteus maximus and medius, biceps femoris, rectus femoris, gastrocnemius, and tibialis anterior compared to when walking without using a smartphone. In summary, the flexed head during walking while using a smartphone lowers the center of gravity, activates more muscles of the neck (Yoon et al., 2020) and lumbar area (Choi et al., 2021), and lessens the activation of the extensor muscle in the lower extremity. Muscle activity imposes less load to the less active muscles and greater load to more active muscles (Kouzaki and Shinohara, 2006). Consequently, the changes in muscle activity during walking associated with smartphone use may undermine functional walking in common pedestrian environments. The frequent use of a smartphone during walking could be a risk factor for musculoskeletal disorders.

There are some limitations to be noted. First, the participants of this study only walked for a short distance in a controlled laboratory space under controlled walking conditions. Second, we only evaluated young students in their 20s and not all ages. Third, the sample size of this study was too small for generalization. Future study should include enough walking distance and many people to cover various situations of smartphone use.

\section{CONFLICT OF INTEREST}

No potential conflict of interest relevant to this article was reported.

\section{ACKNOWLEDGMENTS}

The authors received no financial support for this article.

\section{REFERENCES}

Abraham A, Sommerhalder K, Abel T. Landscape and well-being: A scoping study on the health-promoting impact of outdoor environments. Int J Public Health 2010;55:59-69.

Al-Hayani A. The functional anatomy of hip abductors. Folia Morphol 2009;68:98-103.

Alghamdi W, Preece SJ. How does normal variability in trunk flexion affect lower limb muscle activity during walking? Hum Mov Sci 2020; 72:102630.

Alosaimi FD, Alyahya H, Alshahwan H, Al Mahyijari N, Shaik SA. Smartphone addiction among university students in Riyadh, Saudi Arabia. Saudi Med J 2016;37:675.

Anderson FC, Pandy MG. Individual muscle contributions to support in normal walking. Gait Posture 2003;17:159-169.

Barr AE, Barbe MF, Clark BD. Work-related musculoskeletal disorders of the hand and wrist: epidemiology, pathophysiology, and sensorimotor changes. J Orthop Sports Phys Ther 2004;34:610-627.

Basch CH, Ethan D, Zybert P, Basch CE. Pedestrian behavior at five dangerous and busy Manhattan intersections. J Community Health 2015; 40:789-792.

Berolo S, Wells RP, Amick BC. Musculoskeletal symptoms among mobile hand-held device users and their relationship to device use: a preliminary study in a Canadian university population. Appl Ergon 2011;42: 371-378.

Betsch M, Kalbhen K, Michalik R, Schenker H, Gatz M, Quack V, Migliorini F. The influence of smartphone use on spinal posture - A laboratory study. Gait Posture 2021;85:298-303.

Byington KW, Schwebel DC. Effects of mobile Internet use on college student pedestrian injury risk. Accid Anal Prev 2013;51:78-83.

Choi S, Kim M, Kim E, Shin G. Changes in low back muscle activity and spine kinematics in response to smartphone use during walking. Spine (Phila Pa 1976) 2021;46:E426-E432.

Cronin NJ, Peltonen J, Sinkjaer T, Avela J. Neural compensation within the human triceps surae during prolonged walking. J Neurophysiol 2011;105:548-553.

Douglas EC, Gallagher KM. The influence of a semi-reclined seated posture on head and neck kinematics and muscle activity while reading a tablet computer. Appl Ergon 2017;60:342-347.

Flack NAMS, Nicholson HD, Woodley SJ. A review of the anatomy of the hip abductor muscles, gluteus medius, gluteus minimus, and tensor 
fascia lata. Clin Anat 2012;25:697-708

Gottschalk F, Kourosh S, Leveau B. The functional anatomy of tensor fasciae latae and gluteus medius and minimus. J Anat 1989;166:179-189.

Gustafsson E, Johnson PW, Hagberg M. Thumb postures and physical loads during mobile phone use - A comparison of young adults with and without musculoskeletal symptoms. J Electromyogr Kinesiol 2010; 20:127-135.

Han H, Shin G. Head flexion angle when web-browsing and texting using a smartphone while walking. Appl Ergon 2019;81:102884.

Haxton HA. Absolute muscle force in the ankle flexors of man. J Physiol 1944;103:267-273.

Hermens HJ, Freriks B, Disselhorst-Klug C, Rau G. Development of recommendations for SEMG sensors and sensor placement procedures. J Electromyogr Kinesiol 2000;10:361-374.

Hof AL, Elzinga H, Grimmius W, Halbertsma JPK. Speed dependence of averaged EMG profiles in walking. Gait Posture 2002;16:78-86.

Kepple TM, Siegel KL, Stanhope SJ. Relative contributions of the lower extremity joint moments to forward progression and support during gait. Gait Posture 1997;6:1-8.

Kouzaki M, Shinohara M. The frequency of alternate muscle activity is associated with the attenuation in muscle fatigue. Appl Physiol 2006; 101:715-720.

Lamberg EM, Muratori LM. Cell phones change the way we walk. Gait Posture 2012;35:688-690.

Lee S, Kang H, Shin G. Head flexion angle while using a smartphone. Ergonomics 2015;58:220-226.

Lehman GJ, McGill SM. The importance of normalization in the interpretation of surface electromyography: a proof of principle. J Manipulative Physiol Ther 1999;22:444-446.

Lewton KL, Scott JE. Ischial form as an indicator of bipedal kinematics in early hominins: A test using extant anthropoids. Anat Rec (Hoboken) 2017;300:845-858

Lichtwark GA, Wilson AM. Optimal muscle fascicle length and tendon stiffness for maximising gastrocnemius efficiency during human walking and running. J Theor Biol. 2008;252:662-673.

Lin MIB, Huang YP. The impact of walking while using a smartphone on pedestrians' awareness of roadside events. Accid Anal Prev 2017;101: 87-96.

Liu MQ, Anderson FC, Pandy MG, Delp SL. Muscles that support the body also modulate forward progression during walking. J Biomech 2006; 39:2623-2630.

Liu MQ, Anderson FC, Schwartz MH, Delp SL. Muscle contributions to support and progression over a range of walking speeds. J Biomech 2008;41:3243-3252.

Lovejoy CO. Evolution of human walking. Sci Am 1988;259:118-125.
Megna M, Gisonni P, Napolitano M, Orabona GDA, Patruno C, Ayala F, Balato N. The effect of smartphone addiction on hand joints in psoriatic patients: an ultrasound-based study. J Eur Acad Dermatol Venereol 2018;32:73-78

Ming Z, Pietikainen S, Hänninen O. Excessive texting in pathophysiology of first carpometacarpal joint arthritis. Pathophysiology 2006;13:269270.

Nadeau S, Gravel D, Arsenault AB, Bourbonnais D. Plantarflexor weakness as a limiting factor of gait speed in stroke subjects and the compensating role of hip flexors. Clin Biomech 1999;14:125-135.

Nasar JL, Troyer D. Pedestrian injuries due to mobile phone use in public places. Accid Anal Prev 2013;57:91-95.

Neptune RR, Kautz SA, Zajac FE. Contributions of the individual ankle plantar flexors to support, forward progression and swing initiation during walking. J Biomech 2001;34:1387-1398.

Neptune RR, Sasaki K, Kautz SA. The effect of walking speed on muscle function and mechanical energetics. Gait Posture 2008;28:135-143.

Neptune RR, Zajac FE, Kautz SA. Muscle force redistributes segmental power for body progression during walking. Gait Posture 2004:19:194205.

Pandy MG. Computer modeling and simulation of human movement. Annu Rev Biomed Eng 2001;3:245-273.

Park D, Seong YJ, Woo H, Yoo B, Shim D, Kim ES, Rha DW. Paralysis of the gastrocnemius medial head differentially affects gait patterns and muscle activity during level and stair ascent locomotion. Gait Posture 2019;72:222-227.

Parr ND, Hass CJ, Tillman MD. Cellular phone texting impairs gait in able-bodied young adults. J App Biomech 2014;30:685-688.

Pontzer H, Raichlen DA, Sockol MD. The metabolic cost of walking in humans, chimpanzees, and early hominins. J Hum Evol 2009;56:43-54.

Rubinstein JS, Meyer DE, Evans JE. Executive control of cognitive processes in task switching. J Exp Psychol Hum Percept Perform 2001;27:763797.

Schabrun SM, van den Hoorn W, Moorcroft A, Greenland C, Hodges PW. Texting and walking: strategies for postural control and implications for safety. PLoS One 2014;9:e84312.

Sockol MD, Raichlen DA, Pontzer H. Chimpanzee locomotor energetics and the origin of human bipedalism. Proc Natl Acad Sci U S A 2007; 104:12265-12269.

St George RJ, Fitzpatrick RC. The sense of self-motion, orientation and balance explored by vestibular stimulation. J Physiol 2011;589:807-813.

Stern JT, Jr, Susman RL. The locomotor anatomy of Australopithecus afarensis. Am J Phys Anthropol 1983;60:279-317.

Straker LM, Coleman J, Skoss R, Maslen BA, Burgess-Limerick R, Pollock $\mathrm{CM}$. A comparison of posture and muscle activity during tablet com- 
puter, desktop computer and paper use by young children. Ergonomics 2008;51:540-555.

Usherwood JR, Channon AJ, Myatt JP, Rankin JW, Hubel TY. The human foot and heel-sole-toe walking strategy: a mechanism enabling an inverted pendular gait with low isometric muscle force? J R Soc Interface 2012;9:2396-2402.

Ward SR, Eng CM, Smallwood LH, Lieber RL. Are current measurements of lower extremity muscle architecture accurate? Clin Orthop Relat Res 2009;467:1074-1082.
Winter DA. Overall principle of lower limb support during stance phase of gait. J Biomech 1980;13:923-927.

Yoon W, Choi S, Han H, Shin G. Neck muscular load when using a smartphone while sitting, standing, and walking. Hum Factors 2020 Feb 11; 18720820904237. https://doi.org/10.1177/0018720820904237. Online ahead of print.

Young JG, Trudeau M, Odell D, Marinelli K, Dennerlein JT. Touch-screen tablet user configurations and case-supported tilt affect head and neck flexion angles. Work 2012;41:81-91. 